\title{
Ideal Magnetic Dipole Scattering
}

\author{
Tianhua Feng, ${ }^{1}$ Yi Xu, ${ }^{1,2, *}$ Wei Zhang, ${ }^{1}$ and Andrey E. Miroshnichenko ${ }^{3, \dagger}$ \\ ${ }^{1}$ Department of Electronic Engineering, \\ College of Information Science and Technology, Jinan University, Guangzhou 510632, China \\ ${ }^{2}$ Key Laboratory of Optoelectronic Information and Sensing Technologies of Guangdong Higher Education Institutes, \\ Guangzhou, Guangdong 510632, China \\ ${ }^{3}$ Nonlinear Physics Centre, Research School of Science and Engineering, Australian National University, \\ Acton, Australian Capital Territory 2601, Australia
}

(Received 17 January 2017; published 25 April 2017)

\begin{abstract}
We introduce the concept of tunable ideal magnetic dipole scattering, where a nonmagnetic nanoparticle scatters light as a pure magnetic dipole. High refractive index subwavelength nanoparticles usually support both electric and magnetic dipole responses. Thus, to achieve ideal magnetic dipole scattering one has to suppress the electric dipole response. Such a possibility was recently demonstrated for the so-called anapole mode, which is associated with zero electric dipole scattering. By spectrally overlapping the magnetic dipole resonance with the anapole mode, we achieve ideal magnetic dipole scattering in the far field with tunable strong scattering resonances in the near infrared spectrum. We demonstrate that such a condition can be realized at least for two subwavelength geometries. One of them is a core-shell nanosphere consisting of a Au core and silicon shell. It can be also achieved in other geometries, including nanodisks, which are compatible with current nanofabrication technology.
\end{abstract}

DOI: 10.1103/PhysRevLett.118.173901

Light-matter interaction mainly relies on the electric component of light, especially for nonmagnetic materials [1-6]. The responses of such materials to the magnetic component of light are usually negligible at frequencies above a few terahertz [7-9]. For example, the electromagnetic plane wave scattering of a conducting loop with its dimension much smaller than the wavelength will be dominated by its electric response. Therefore, the realization of effective optical magnetism is very crucial for designing functional metamaterials working at this spectrum range [10-12]. Optical Mie resonances in dielectric nanoparticles with high refractive indices provide a platform for studying magnetic light-matter interaction mediated by their optical induced magnetic resonances [13-16]. Such a nanoparticle supports a magnetic dipole (MD) mode, which resembles a circular displacement current distribution in the near field, and therefore manifests itself as a MD scatter in the far field. To date, various promising functions have been demonstrated via involving magnetic resonances, such as directional scattering of light [17-25], Fano resonances [26-29], enhanced optical nonlinearity [30], nonradiate anapole [31], subwavelength topological edge states [32], Purcell factor enhancements [33-36], and metasurface $[37,38]$ etc.

In general, the electric dipole (ED) and MD modes in silicon nanoparticles (SNs) suffer from spectral overlapping, as shown by many examples in a recently published review paper [13]. Therefore, a $\mathrm{SN}$ is indeed a "dressed" MD scatter [13,15,16,31]. They can exhibit various interference effects in the far field, leading to the unidirectional Fano resonant scattering of light [18-20].
For unambiguously studying the MD scattering, it is necessary to suppress the ED and all higher-order multipoles. Such kind of exotic ideal MD scattering would be extremely useful for the unambiguous study of magnetic light-matter interaction in nonmagnetic nanoparticles.

Regarding a SN, the conditions to realize the ideal MD scattering are the solutions of the following sets of inequalities, corresponding to the condition of resonant overlap between the anapole and MD modes as well as negligible contributions to the total scattering from higherorder multipolar modes (details can be found in the Supplemental Material [39]):

$$
\begin{aligned}
\left|\left(D_{1}(m k R) / m+1 /(k R)\right) \psi_{1}(k R)-\psi_{0}(k R)\right| & \ll 1 \\
{\left[\left|b_{1}(\lambda)\right|_{R}\right]^{\prime}=0, \quad\left[\left|b_{1}(\lambda)\right|_{R}\right]^{\prime \prime} } & <0 \\
\left|a_{n}(\lambda, R)\right| \ll 1, \quad\left|b_{n}(\lambda, R)\right| \ll 1, \quad n & >1,
\end{aligned}
$$

where $k=2 \pi / \lambda$ is the free space wave number, $\lambda$ is the wavelength in vacuum, $R$ is the radius of the $\mathrm{SN}, m$ is the relative refractive index of silicon $(\mathrm{Si})$ with respect to the ambient medium, $D_{n}(m z)=\psi_{n}^{\prime}(m z) / \psi_{n}(m z), \psi_{n}(z)$ is the Riccati-Bessel function, and $a_{n}, b_{n}$ are the Mie scattering coefficients. The prime means derivative with respect to the argument in parentheses after obtaining the absolute value of the function. Thus, we have just two free parameters-incident wavelength $\lambda$ and radius $R$. However, one can easily check that these sets of inequalities do not have a solution for $\lambda$ and $R$ in the visible and near infrared wavelength range. It can be understood due to the fact that these two parameters are related to each other via the 
so-called size parameter $q=2 \pi R / \lambda$ and simply changing the radius of SN will simultaneously shift the resonances of the ED and MD along the same spectrum direction while the spectrum overlap between the ED and MD is always preserved $[15,16]$. But, in general, there is a possibility to achieve ideal MD scattering via overlapping the MD mode with the nonradiated anapole mode that had been demonstrated very recently in SNs [31]. It has also been proved that such an anapole can be effectively tuned in $\mathrm{Ag}$ core-Si shell nanoparticle to realize scattering transparency [40]. Therefore, it is expected that core-shell nanoparticles could provide the possibility to suppress the ED mode at the MD resonance.

In this Letter, we introduce the concept of ideal $M D$ scattering whose ED and high order electric and magnetic multipoles are negligible at the resonant frequency of a MD mode. Such suppression is based on the excitation of the anapole mode, which is a result of the destructive interference between Cartesian ED and toroidal dipole (TD) modes $[31,40]$, in a Au core-Si shell nanoparticle. We show that there are two ED modes in this hybrid system, and their interaction can be used to shift the resonant frequency of the anapole mode to overlap with the MD mode. We demonstrate both analytically and numerically that the total scattering of a Au core-Si shell nanoparticle is completely dominated by its MD mode, which manifests itself as an ideal MD scatter based on nonmagnetic materials.

As mentioned above, the spectral overlap of ED and MD modes is always presented. In Fig. 1(a), we show the results based on the Mie theory [41] for a $\mathrm{SN}$ of radius $R=180 \mathrm{~nm}$. The MD resonance occurs when the size of a SN satisfies the condition $\lambda_{0} / n_{S i} \approx 2 R_{S i}$ [14-16], where $R_{S i}$ is the radius of the $\mathrm{SN}, n_{S i}$ is the refractive index of $\mathrm{Si}$ and $\lambda_{0}$ is the free space wavelength. Considerable spectrum overlap between ED and MD modes in the range 900$1600 \mathrm{~nm}$ is observed. It can be seen from Figs. 1(b) and 1(c) that the far-field scattering pattern at the resonant frequency of the MD is asymmetric and deviates from an ideal dipole type scattering pattern with nonzero scattering intensities along the MD axis at $90^{\circ}$ and $270^{\circ}$. It means that the ED component contributes to the far-field scattering, and the SN cannot be regarded as an ideal MD scatter.

For a typical Au core-Si shell nanoparticle, the degree of spectrum overlap between the ED and MD resonances can be reduced (see Fig. S1 in the Supplemental Material [39]). Compared with the SN of the same outer radius shown in Fig. 1, the MD resonant wavelength is almost unchanged, and the MD contribution to the total scattering is only slightly reduced as the $\mathrm{Au}$ core is located at the node of the electric field for the MD mode. Most importantly, the anapole mode appears around $\sim \lambda=1.25 \mu \mathrm{m}$ (see Fig. S1 in the Supplemental Material [39]). These results suggest that there is a possibility to overlap the anapole and MD modes in core-shell nanoparticles. Analytically, the conditions to obtain the ideal MD scattering of a core-shell

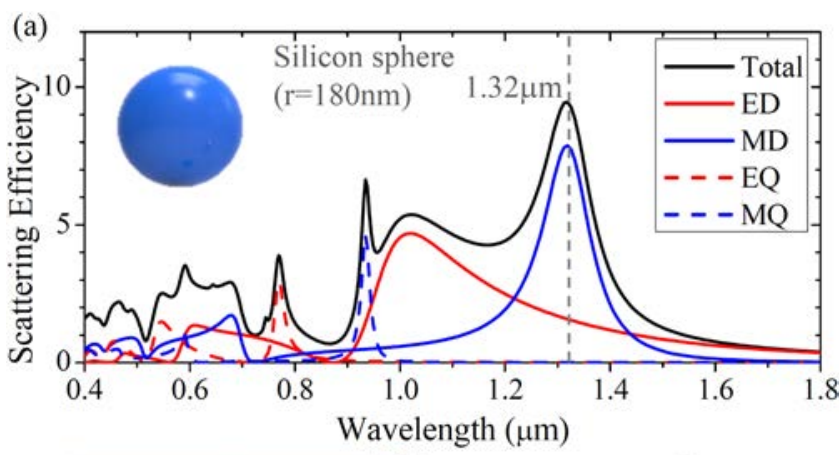

(b)

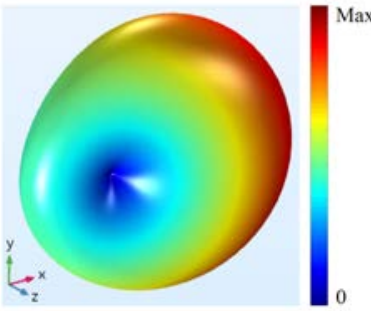

(c)

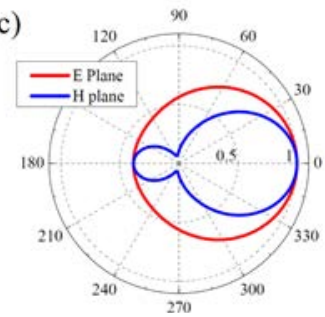

FIG. 1. (a) Scattering efficiency of a Si sphere embedded in air with a radius $R$ of $180 \mathrm{~nm}$. The contributions of the spherical ED, $\mathrm{MD}$, electric quadrupole (EQ), and magnetic quadrupole (MQ) to the total scattering are presented. (b) A far-field scattering pattern at the MD resonant wavelength marked by a dashed line in (a). A plane wave is impinged along the $x$ axis with its electric polarization along the $y$ axis. (c) Cross section views on the $x-y$ plane ( $E$ plane) and $x-z$ plane ( $H$ plane) of the far-field scattering pattern shown in (b).

nanoparticle are the solutions of the following sets of inequalities:

$$
\begin{aligned}
\left|\left(\tilde{D}_{1} / m_{2}+1 /(k R)\right) \psi_{1}(k R)-\psi_{0}(k R)\right| & \ll 1 \\
{\left[\left|b_{1}(\lambda)\right|_{r, R}\right]^{\prime}=0, \quad\left[\left|b_{1}(\lambda)\right|_{r, R}\right]^{\prime \prime} } & <0, \\
\left|a_{n}(\lambda, r, R)\right| \ll 1, \quad\left|b_{n}(\lambda, r, R)\right| \ll 1, \quad n & >1,
\end{aligned}
$$

with

$$
\begin{aligned}
& \tilde{D}_{1}=\frac{D_{1}\left(m_{2} k R\right)-A_{1} \chi_{1}^{\prime}\left(m_{2} k R\right) / \psi_{1}\left(m_{2} k R\right)}{1-A_{1} \chi_{1}\left(m_{2} k R\right) / \psi_{1}\left(m_{2} k R\right)} \\
& \tilde{G}_{1}=\frac{D_{1}\left(m_{2} k R\right)-B_{1} \chi_{1}^{\prime}\left(m_{2} k R\right) / \psi_{1}\left(m_{2} k R\right)}{1-B_{1} \chi_{1}\left(m_{2} k R\right) / \psi_{1}\left(m_{2} k R\right)} \\
& A_{1}=\frac{\psi_{1}\left(m_{2} k r\right)\left(m D_{1}\left(m_{1} k r\right)-D_{1}\left(m_{2} k r\right)\right)}{m D_{1}\left(m_{1} k r\right) \chi_{1}\left(m_{2} k r\right)-\chi_{1}^{\prime}\left(m_{2} k r\right)} \\
& B_{1}=\frac{\psi_{1}\left(m_{2} k r\right)\left(D_{1}\left(m_{1} k r\right) / m-D_{1}\left(m_{2} k r\right)\right)}{D_{1}\left(m_{1} k r\right) \chi_{1}\left(m_{2} k r\right) / m-\chi_{1}^{\prime}\left(m_{2} k r\right)},
\end{aligned}
$$

where $k=2 \pi / \lambda$ is the free space wave number, $\lambda$ is the wavelength in vacuum, $r$ and $R$ are the inner and outer radius of the core-shell nanoparticle, and $m=m_{2} / m_{1}$ with 
$m_{1}$ and $m_{2}$ are the relative refractive indices of inner and outer materials relative to the ambient medium.

One set of the typical solutions for the sets of inequalities (2) in the optical spectrum range, $600 \mathrm{~nm}<\lambda<1600 \mathrm{~nm}$, are $\lambda=1280 \mathrm{~nm}, \quad r=62 \mathrm{~nm}$, and $R=180 \mathrm{~nm}$ (see Fig. S2 in the Supplemental Material [39] for other solutions). Figure 2(a) presents the results for this case. One can see that the spherical ED contribution vanishes exactly at the spherical MD resonance. Meanwhile, the contributions from EQ and MQ modes are negligible. The far-field scattering results shown in Figs. 2(a) and 2(b) also validate a pure MD response, exhibiting as a doughnut shape and zero scattering intensities along the $\mathrm{MD}$ axis $\left(90^{\circ}\right.$ and $\left.270^{\circ}\right)$. For comparison, we also provide the results at the peak of the ED mode in Figs. 2(a) and 2(c). They obviously deviate from the ideal ED scattering because of the spectrum overlap between ED and MD modes.
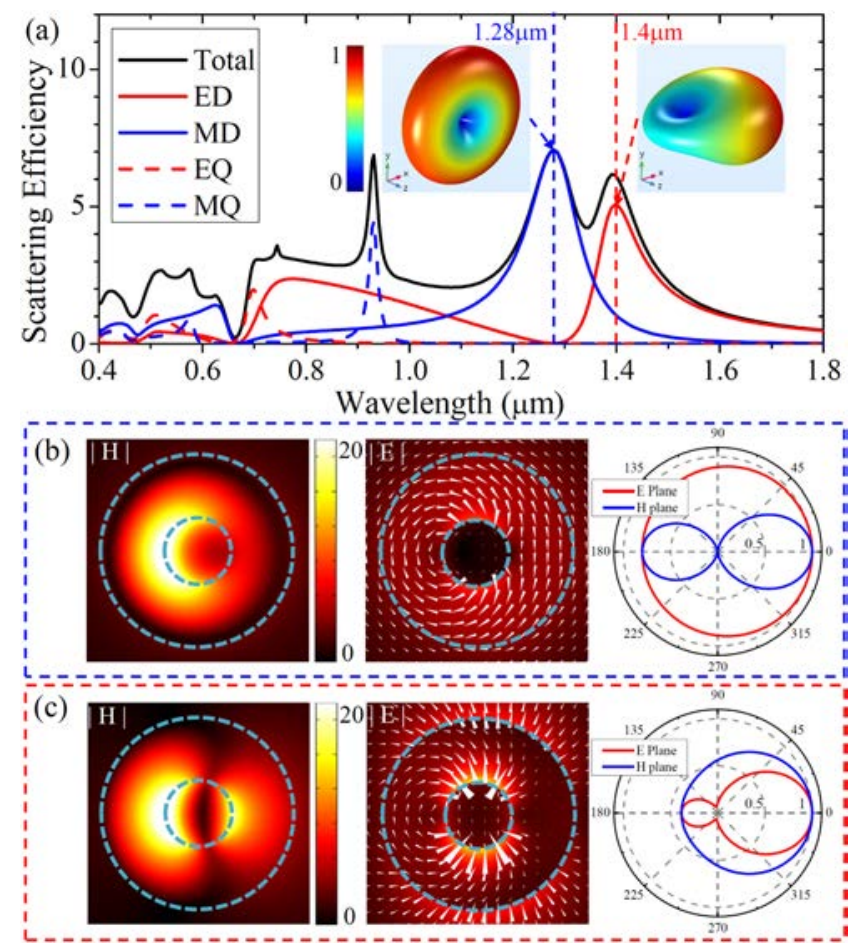

FIG. 2. (a) Scattering efficiency of a Au core-Si shell nanoparticle embedded in air with an outer radius $R=180 \mathrm{~nm}$ and an inner radius $r=62 \mathrm{~nm}$. The contributions of the spherical ED, $\mathrm{MD}, \mathrm{EQ}$, and MQ to the total scattering are also presented. A plane wave is incident along the $x$ axis with its electric polarization along the $y$ axis. The far-field scattering patterns at the MD and ED resonant wavelengths indicated by dashed lines are presented in the insets. The induced electric $|E|$ and magnetic $|H|$ near-field distributions, which are obtained by subtracting the incident field from the total field, are shown for MD (b) and ED (c) modes. The corresponding cross section views of the far-field scattering patterns in (a) are also shown in (b) and (c).
In order to further explore the underlying physics for the overlapping of the anapole and MD modes in a coreshell nanoparticle, we inspect the scattering of a $\mathrm{Au}$ nanoparticle embedded in a Si background first, as shown in Figs. 3(a)-3(c). As expected, there is no MD response for the Au particle [see Fig. 3(a)]. The dependence of the contribution of the spherical ED to the total scattering on the size of the Au nanoparticle is shown in Fig. 3(b). One can see that there is a lower limit for the resonant wavelength of the ED mode $(\lambda=810 \mathrm{~nm})$. We further consider the case of a $\mathrm{Si}$ shell $(R=180 \mathrm{~nm})$ embedded in vacuum with a varied size of an air core (void) in Figs. 3(d)-3(f). As can be seen from these figures, $\mathrm{ED}$ and MD resonances are blueshifted together with the increasing of the void size due to the reduction of the effective permittivity. Finally, we inspect the case of a $\mathrm{Au}$ core-Si shell nanoparticle shown in Figs. 3(g)-3(i). It is shown that there is a bifurcation point in the spherical ED decomposition results when we increase the radius $r$ of a Au core from $10 \mathrm{~nm}$ to $25 \mathrm{~nm}$ [see Figs. 3(h) and 3(i)], indicating strong interaction between two ED modes and the effective excitation of the anapole mode (destructive interference between Cartesian ED and TD modes) [31]. Most importantly, we can conclude from Figs. 3(h) and 3(i) that the anapole mode can be fine-tuned from 900-1600 nm, which can resonantly overlap with the MD mode at the optical spectrum shown in Fig. 3(g).

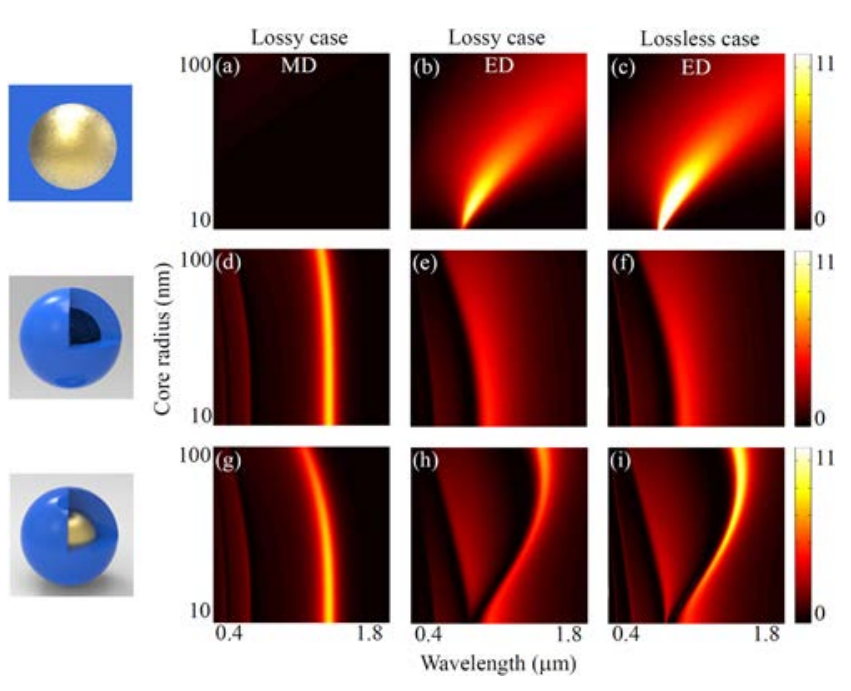

FIG. 3. The first column: Sketches of various nanoparticle geometries, which show the Au nanosphere embedded in $\mathrm{Si}, \mathrm{Si}$ shell with a void, and Au core-Si shell nanoparticle embedded in air. The outer radius $R$ of Si shells is $180 \mathrm{~nm}$; the second column: the corresponding spherical MD contributions to the total scattering referring to the structure on the left; the third column: the corresponding spherical ED contributions to the total scattering for the lossy case; the fourth column: the corresponding spherical ED contributions to the total scattering for the lossless case. 
In order to validate the effective excitation of the anapole mode in this nanoparticle, we calculate the scattering efficiency of the Cartesian ED and TD as [42-45]

$$
\sigma_{P}=\frac{\mu_{0} \omega^{4}}{12 \pi^{2} R^{2} c}|\mathbf{P}|^{2}, \quad \sigma_{T}=\frac{\mu_{0} \omega^{4} k^{2}}{12 \pi^{2} R^{2} c}|\mathbf{T}|^{2},
$$

where

$$
\begin{gathered}
\mathbf{P}=\frac{1}{-i \omega} \int d^{3} r \mathbf{J}(\mathbf{r}) \\
\mathbf{T}=\frac{1}{10 c} \int d^{3} r\left[(\mathbf{r} \cdot \mathbf{J}(\mathbf{r})) \mathbf{r}-2 r^{2} \mathbf{J}(\mathbf{r})\right] .
\end{gathered}
$$

$\mu_{0}$ is the permeability of vacuum, $\omega$ is the angular frequency, $c$ is the speed of light, and $\mathbf{r}$ specifies the location where the induced current $\mathbf{J}$ is evaluated. As can be seen in Fig. 4, there is a cross point for the scattering efficiency of the Cartesian ED and TD contributions, implying that the condition $(\mathbf{P}=-i k \mathbf{T})$ when the Cartesian ED and TD can cancel each other in the far field has been fulfilled [31,40]. The slight difference between the wavelengths where the contribution of the Cartesian ED is equal to the TD and the minimum response of the spherical ED is due to the material losses. Figure 4(b) presents the lossless case in which the crossing point of the Cartesian ED and TD is exactly at the same wavelength as that of the spherical ED with zero response, indicating the excitation of the anapole mode at the MD resonance, i.e.,
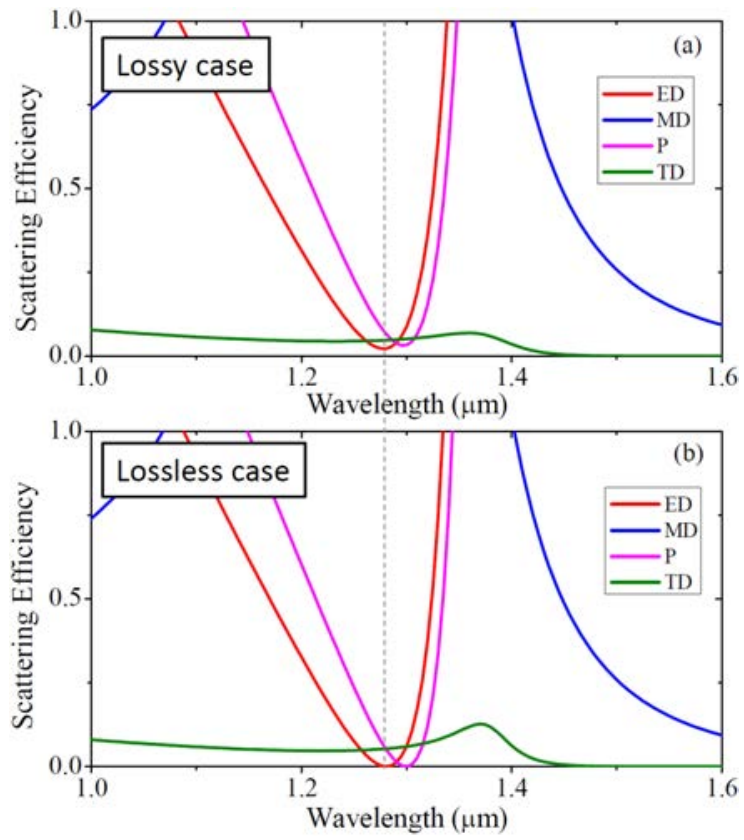

FIG. 4. Spherical ED (red) and MD (blue), Cartesian ED (P, purple) and TD (green) moments contributions to the total scattering of the core-shell nanoparticle shown in Fig. 3 for lossy (a) and lossless (b) cases. Vertical dashed line indicates the resonant frequency, where the ideal MD scattering appears. the ideal MD scattering. It should be noted here that we only mimic the pure MD scattering in the far field, since the near-field distribution of electromagnetic fields shown in Fig. 2(b) is a superposition of MD and anapole modes. Since a planar nanostructure is more compatible with the state-of-the-art nanofabrication technologies for Si photonics, a planar ideal magnetic scatter is of great demand compared with the spherical core-shell nanoparticle. We further provide a design for ideal MD scattering based on a nanodisk. The plane wave scattering results of the Au core$\mathrm{Si}$ shell nanodisk shown in Fig. 5 confirm that such a structure can also realize the ideal MD scattering in the optical wavelength range (see Fig. S3 in the Supplemental Material [39] for the decomposition results). We also investigate the effect of imperfections on the ideal MD scattering. The relative broadband dipolar resonance we used might release the fabrication restriction of an experimental demonstration. We model the imperfections in the fabrication process with a $5 \mathrm{~nm}$ position variation of the $\mathrm{Au}$ core according to the state-of-the-art fabrication technology. The calculated results demonstrate that the proposed ideal MD scattering is still robust when moderate fabrication imperfections are taken into account (see Fig. S4 in the Supplemental Material [39]).

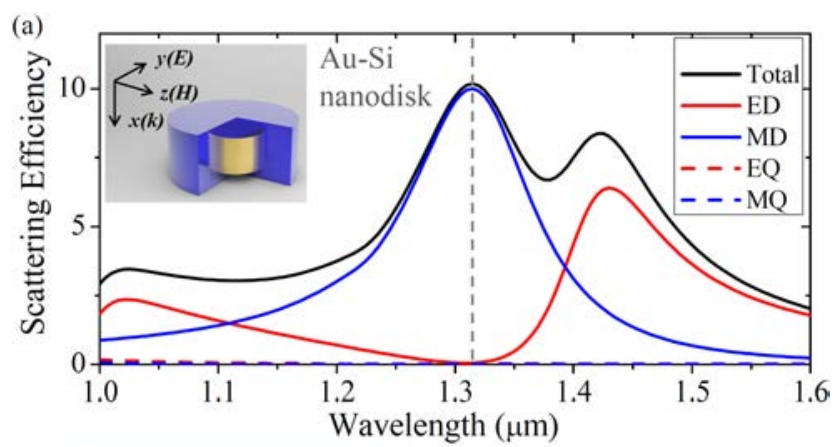

(b)

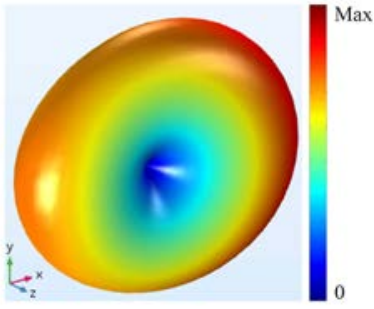

(c)

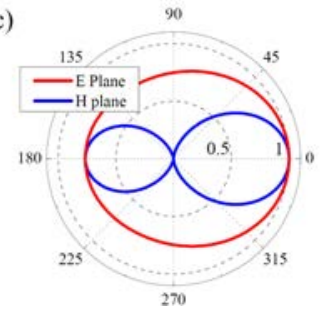

FIG. 5. (a) Scattering efficiency of a Au core-Si shell nanodisk embedded in air with the outer radius $R=160 \mathrm{~nm}$, outer height $H=350 \mathrm{~nm}$, and an inner radius $r=50 \mathrm{~nm}$, inner height $h=125 \mathrm{~nm}$. The contributions of the spherical ED, MD, EQ, and MQ to the total scattering are also presented based on the decomposition utilizing spherical harmonics [46]. A schematic shows the geometry of the nanodisk. A plane wave is incident along the axis of the cylinder ( $x$ axis) with its electric polarization along the $y$ axis. (b) The far-field scattering pattern at the ideal MD scattering wavelength indicated by the dashed line in (a).

(c) Cross section views on the $x-y$ plane ( $E$ plane) and $x-z$ plane ( $H$ plane) of the far-field scattering pattern shown in (b). 
It should be stressed that the radiative damping of the scattering process that surpasses the dissipative losses is crucial to realize ideal MD scattering, similar to the condition of anomalous light scattering by small particles [2]. The ideal MD scattering can also be realized in the visible spectrum utilizing a dielectric material with a moderate-refractive-index as a shell [47] (see Fig. S5 in the Supplemental Material [39]). Our results demonstrate that structured nonmagnetic nanoparticles can be used to realize the ideal $M D$ scattering. It should be pointed out that structured light can also be used to realize the ideal MD scattering as the MD mode in Si nanoparticle resembles the electromagnetic field distributions for an azimuthally polarized beam $[36,48]$.

In summary, we demonstrate both analytically and numerically that $\mathrm{Au}$ core-Si shell nanoparticles, including spherical and disk geometries, can be used to realize the ideal MD scattering, where other multipole modes (including the ED mode) are suppressed, with tunable resonant wavelengths up to the visible range. These types of nanoparticles provide an ideal platform to study the magnetic light-matter interaction in nanoscale, such as the tailoring of MD transition. We expect that functional magnetic light based devices can be realized, such as a pure magnetic mirror and ideal directional magnetic scattering. Our results might also facilitate the emergence of a new subfield in the magnetic light-matter interaction based on nonmagnetic materials.

Y. Xu thanks the financial support from National Natural Science Foundation of China (Grants No. 11674130 and No. 11304047); Natural Science Foundation of Guangdong Province, China (Grants No. 2016A030306016, No. 2014 A030313376).

*e_chui@qq.com

†andrey.miroshnichenko@anu.edu.au

[1] A. F. Koenderink, M. Kafesaki, B. C. Buchler, and V. Sandoghdar, Phys. Rev. Lett. 95, 153904 (2005).

[2] M. I. Tribelsky and B. S. Luk'yanchuk, Phys. Rev. Lett. 97, 263902 (2006).

[3] M. Burresi, D. van Oosten, T. Kampfrath, H. Schoenmaker, R. Heideman, A. Leinse, and L. Kuipers, Science 326, 550 (2009).

[4] Z. C. Ruan and S. H. Fan, Phys. Rev. Lett. 105, 013901 (2010).

[5] L. Verslegers, Z. F. Yu, Z. C. Ruan, P. B. Catrysse, and S. F. Fan, Phys. Rev. Lett. 108, 083902 (2012).

[6] N. Rotenberg and L. Kuipers, Nat. Photonics 8, 919 (2014).

[7] L. D. Landau and E. M. Lifshitz, Electrodynamics of Continuous Media (Wiley, New York, 1984).

[8] M. Burresi, T. Kampfrath, D. van Oosten, J. C. Prangsma, B. S. Song, S. Noda, and L. Kuipers, Phys. Rev. Lett. 105, 123901 (2010).
[9] S. Vignolini, F. Intonti, F. Riboli, L. Balet, L. H. Li, M. Francardi, A. Gerardino, A. Fiore, D. S. Wiersma, and M. Gurioli, Phys. Rev. Lett. 105, 123902 (2010).

[10] J. C. Ginn, I. Brener, D. W. Peters, J. R. Wendt, J. O. Stevens, P. F. Hines, L. I. Basilio, L. K. Warne, J. F. Ihlefeld, P. G. Clem, and M. B. Sinclair, Phys. Rev. Lett. 108, 097402 (2012).

[11] S. Jahani and Z. Jacob, Nat. Nanotechnol. 11, 23 (2016).

[12] M. Kasperczyk, S. Person, D. Ananias, L. D. Carlos, and L. Novotny, Phys. Rev. Lett. 114, 163903 (2015).

[13] A. I. Kuznetsov, A. E. Miroshnichenko, M. L. Brongersma, Y. S. Kivshar, and B. S. Luk'yanchuk, Science 354, 2472 (2016).

[14] A. García-Etxarri, R. Gómez-Medina, L. S. Froufe-Pérez, C. López, L. Chantada, F. Scheffold, J. Aizpurua, M. NietoVesperinas, and J. J. Sáenz, Opt. Express 19, 4815 (2011).

[15] A. I. Kuznetsov, A. E. Miroshnichenko, Y. H. Fu, J. Zhang, and B. S. Luk'yanchuk, Sci. Rep. 2, 492 (2012).

[16] A. B. Evlyukhin, S. M. Novikov, U. Zywietz, R. L. Eriksen, C. Reinhardt, S. I. Bozhevolnyi, and B. N. Chichkov, Nano Lett. 12, 3749 (2012).

[17] Y. G. Liu, W. C. H. Choy, W. E. I. Sha, and W. C. Chew, Opt. Lett. 37, 2112 (2012).

[18] W. Liu, A. E. Miroshnichenko, D. N. Neshev, and Y. S. Kivshar, Phys. Rev. B 86, 081407 (2012).

[19] Y. H. Fu, A. I. Kuznetsov, A. E. Miroshnichenko, Y. F. Yu, and B. S. Luk'yanchuk, Nat. Commun. 4, 1527 (2013).

[20] S. Person, M. Jain, Z. Lapin, J. J. Sáenz, G. Wicks, and L. Novotny, Nano Lett. 13, 1806 (2013).

[21] I. Staude, A. E. Miroshnichenko, M. Decker, N. T. Fofang, S. Liu, E. Gonzales, J. Dominguez, T. S. Luk, D. N. Neshev, I. Brener, and Y. Kivshar, ACS Nano 7, 7824 (2013).

[22] S. V. Li, D. G. Baranov, A. E. Krasnok, and P. A. Belov, Appl. Phys. Lett. 107, 171101 (2015).

[23] Y. Yang, Q. Li, and M. Qiu, Appl. Phys. Lett. 107, 031109 (2015).

[24] H. Wang, P. Liu, Y. Ke, Y. Su, L. Zhang, N. Xu, S. Deng, and H. Chen, ACS Nano 9, 436 (2015).

[25] J. H. Yan, P. Liu, Z. Y. Lin, H. Wang, H. J. Chen, C. X. Wang, and G. W. Yang, Nat. Commun. 6, 7042 (2015).

[26] A. E. Miroshnichenko and Y. S. Kivshar, Nano Lett. 12, 6459 (2012).

[27] K. E. Chong, B. Hopkins, I. Staude, A. E. Miroshnichenko, J. Dominguez, M. Decker, D. N. Neshev, I. Brener, and Y. S. Kivshar, Small 10, 1985 (2014).

[28] J. Yan, P. Liu, Z. Lin, H. Wang, H. Chen, C. Wang, and G. Yang, ACS Nano 9, 2968 (2015).

[29] Y. Tsuchimoto, T. Yano, T. Hayashi, and M. Hara, Opt. Express 24, 14451 (2016).

[30] M. Shcherbakov, D. N. Neshev, B. Hopkins, A. Shorokhov, I Staude, E. Melik-Gaykazyan, M. Decker, A. Ezhov, A. E. Miroshnichenko, I. Brener, and Y. S. Kivshar, Nano Lett. 14, 6488 (2014).

[31] A. E. Miroshnichenko, A. B. Evlyukhin, Y. F. Yu, R. M. Bakker, A. Chipouline, A. I. Kuznetsov, B. S. Luk'yanchuk, B. N. Chichkov, and Y. S. Kivshar, Nat. Commun. 6, 8069 (2015).

[32] A. P. Slobozhanyuk, A. N. Poddubny, A. E. Miroshnichenko, P. A. Belov, and Y. S. Kivshar, Phys. Rev. Lett. 114, 123901 (2015). 
[33] M. K. Schmidt, R. Esteban, J. J. Sáenz, I. Suárez-Lacalle, S. Mackowski, and J. Aizpurua, Opt. Express 20, 13636 (2012).

[34] M. Decker, I. Staude, I. I. Shishkin, K. B. Samusev, P. Parkinson, V. K. A. Sreenivasan, A. Minovich, A. E. Miroshnichenko, A. Zvyagin, C. Jagadish, D. N. Neshev, and Y. S. Kivshar, Nat. Commun. 4, 2949 (2013).

[35] X. Zambrana-Puyalto and N. Bonod, Phys. Rev. B 91, 195422 (2015).

[36] T. H. Feng, Y. Xu, Z. X. Liang, and W. Zhang, Opt. Lett. 41, 5011 (2016).

[37] Y. M. Yang, I. I. Kravchenko, D. P. Briggs, and J. Valentine, Nat. Commun. 5, 5753 (2014).

[38] J. Xiang, J. X. Li, H. Li, C. Y. Zhang, Q. F. Dai, S. L. Tie, and S. Lan, Opt. Express 24, 11420 (2016).

[39] Supplemental Material at http://link.aps.org/supplemental/ 10.1103/PhysRevLett.118.173901, which includes the conditions for the pure magnetic dipole scattering, the plane wave scattering of a $\mathrm{Au}$ core-Si shell nanoparticle, the tunable ideal MD scattering, the origin of anapole mode in a Au core-Si shell nanodisk and the role of imperfections, and the ideal magnetic dipole scattering in the visible spectrum range.

[40] W. Liu, J.F. Zhang, and A.E. Miroshnichenko, Laser Photonics Rev. 9, 564 (2015).

[41] C. F. Bohren and D. R. Huffman, Absorption and Scattering of Light by Small Particles (John Wiley \& Sons, New York, 1983).

[42] E. E. Radescu and G. Vaman, Phys. Rev. E 65, 046609 (2002).

[43] T. Kaelberer, V. A. Fedotov, N. Papasimakis, D. P. Tsai, and N. I. Zheludev, Science 330, 1510 (2010).

[44] V. A. Fedotov, A. V. Rogacheva, V. Savinov, D. P. Tsai, and N. I. Zheludev, Sci. Rep. 3, 2967 (2013).

[45] N. Papasimakis, V. A. Fedotov, V. Savinov, T. A. Raybould, and N. I. Zheludev, Nat. Mater. 15, 263 (2016).

[46] P. Grahn, A. Shevchenko, and M. Kaivola, New J. Phys. 14, 093033 (2012).

[47] S. Zhang, R. Jiang, Y. M. Xie, Q. Ruan, B. Yang, J. Wang, and H. Q. Lin, Adv. Mater. 27, 7432 (2015).

[48] T. Das, P. P. Iyer, R. A. DeCrescent, and J. A. Schuller, Phys. Rev. B 92, 241110(R) (2015). 